\title{
Electrical Charge Transfer in Complex Oxides
}

\author{
V.N. SHEVCHUK* AND I.V. KAYUN \\ Laboratory of Oxide Crystals, Department of Electronics \\ Lviv Ivan Franko National University, Lviv, Ukraine
}

\begin{abstract}
The electrophysical properties (temperature-frequency dependence of conductivity and dielectric constant, current of depolarization) of complex oxide single crystals (Ca-Ga-Ge-garnet, Ca-Ga-germanate, Pb-, Ca-, Cd-tungstates) are analyzed. The experimental results for different crystallographic axes were obtained in the frequency range $10-10^{5} \mathrm{~Hz}$ and temperature range 290-650 K. The main parameters of conductivity and depolarization currents are determined. The nature of polarization charge induced by applied external field is discussed using the model of polarization at hopping exchange of charge between complex dipolar defects and space-inhomogeneous distribution of charge in the sample. Existence of small polarons in the crystals is considered.
\end{abstract}

PACS numbers: 72.20.-i, 72.80.-r, 77.22.Gm, 77.84.-s

\section{Introduction}

In this paper, it is considered the experimental data of study of crystals with different structure types, from the point of view of behaviour of electrically active structural defects in complex oxides under the external electrical field. The electronic part of electrical transfer is investigated. The features of electric and dielectric properties caused partly by complex point defects and their temperature behaviour in $\mathrm{Ca}_{3} \mathrm{Ga}_{2} \mathrm{Ge}_{3} \mathrm{O}_{12}$ and $\mathrm{Ca}_{3} \mathrm{Ga}_{2} \mathrm{Ge}_{4} \mathrm{O}_{14}$ single crystals (with the garnet (CGGG) and Ca-gallogermanate (CGG) structure, respectively), and in $\mathrm{AWO}_{4}: \mathrm{PbWO}_{4}(\mathrm{PbWO}), \mathrm{CaWO}_{4}(\mathrm{CaWO})$, and $\mathrm{CdWO}_{4}(\mathrm{CdWO})$ (with the sheelite and volframite structure, respectively) are also considered. In this work, main emphasis is paid to investigation of undoped crystals. In the case of CGGG measurements were carried out also for the crystals containing the introduced $\mathrm{Cr}, \mathrm{Mn}, \mathrm{Co}$, $\mathrm{Nd}$, Er impurities.

The mentioned oxides are well-known compounds as materials for modern science and technology. The CGGG and CGG crystals doped with $\mathrm{Nd}$ are suggested as laser materials [1]. The CGG compound as polyfunctional material is known for its piezoelectric properties and optical activity [2]. The AWO crystals have important optical-luminescent properties [3, 4] and are used in spectroscopy of high-energy charged particles $[5,6]$.

However, the temperature-frequency dynamic of ionelectronic processes in these crystals and their physical properties (especially below $500 \mathrm{~K}$ ) are investigated insufficiently. The united opinions on the models of struc-

* corresponding author; e-mail:

shevchuk@electronics.wups.lviv.ua tural defects in these crystals are absent. Establishing the complex formation regularities in oxide crystals at the moment are at the stage of data accumulation, although the effects of structure defects aggregation, as it was shown in recent investigations $[7,8]$ are typical for all the oxides.

Some electrophysical properties of the nonactivated CGGG [9], CGG [10], AWO [11-13] crystals were previously investigated to ascertain the regularities of electrical transfer in direct applied field. The CGG [10] and AWO [13] crystals were investigated also in alternating field at the frequency $f=1 \mathrm{kHz}$. But, these measurements did not explain the mechanisms of electrical conductivity $\sigma$ and features of temperature behaviour of dielectric characteristics for the crystals under investigation. The nature of temperature anomalous behaviour of the physical properties of the CGG crystal [10] is not established. The discussions on the possible structural transformations and the search for phase transitions in CGG are continued [14]. We connect the thermodielectric effect in PbWO crystals [15], previously excited in electrical field, with the dipolar polarization processes of the vacancy type complex defects which are based on dipolons $[13,16]$. However, the nature of the observed phenomenon of nonlinear behaviour of dielectric constant $\varepsilon$ of the $\mathrm{PbWO}$ crystal at the temperature change in the region 290-380 K needs additional investigations. In the CdWO and CaWO crystals at the same conditions of measurements, the thermodielectric effect was not observed. At the same time, in CdWO the thermally stimulated depolarization (TSD) currents of dipolar nature $[12,13]$ are detected. Such an electrical behaviour of the AWO crystals demands detailed investigations and consideration, with taking into account the features of growth technology and structure of these tungstates. 


\section{Experimental}

The electrotransfer regularity and relaxation processes were established on the basis of temperature-frequency investigations of the real $\sigma^{\prime}$ and imaginary $\sigma^{\prime \prime}$ parts of the complex conductivity

$$
\sigma^{*}=\sigma^{\prime}+\mathrm{i} \sigma^{\prime \prime}
$$

and the dielectric constant accordingly

$$
\varepsilon^{*}=\varepsilon^{\prime}-\mathrm{i} \varepsilon^{\prime \prime}
$$

with taking into account the conductivity $\sigma$ in direct applied electrical field and TSD currents. The investigations are carried out for the CGGG crystals $\left(O_{h}^{10}-I a 3 d\right.$ space group) and CGG of trigonal Ca-gallogermanate structural type $\left(O_{3}^{2}-P 321\right.$ space group). The measurements are carried out parallel to the growth axis [100] for cubic garnet crystals, parallel to the principal axis $z-$ [0001], (z-cut), and perpendicular to the axis $x-y(x-y$ cut) for CGG acentric crystals. With the aim of comparison with the data for undoped CGGG we investigated the CGGG crystals with the introduced impurities of $\mathrm{Cr}$, Co, Mn, Nd (0.02 at.\%), and Er (0.002 at.\%). The measurements on specimens of PbWO and CaWO $\left(C_{4 h}^{6}-I 4_{1} / a\right.$ space group) were performed parallel to the growth axis [001] and perpendicular to the cleavage face (010) of the CdWO crystal $\left(C_{2 h}^{4}-P 2 / c\right.$ space group). The crystals were obtained by the Czochralski method. The specimens were prepared with $10 \times 10 \times 0.4 \mathrm{~mm}^{3}$ dimensions. The aquaaquadag or Ag-paste were used as contacts.

Frequency dependences of dielectric characteristics of crystals are tested in the frequency range $10-10^{5} \mathrm{~Hz}$ as described previously in Ref. [17]. Temperature $T$ in the range $290-650 \mathrm{~K}$ was varied linearly at a rate of $0.1 \mathrm{~K} / \mathrm{s}$ both at heating and cooling. The external applied direct field was not greater than $10^{5} \mathrm{~V} / \mathrm{m}$. The currents of conductivity and TSD ( $\left.I_{\mathrm{TSD}}\right)$ of thermoelectret state were measured by a voltmeter-electrometer of V7-30 type using the procedure described elsewhere $[13,16]$. The nature of polarization in the frame of dipolar or of space-charge formation mechanisms [18] was established by the variation of polarization conditions (polarization voltage $U_{\mathrm{p}}$, polarization temperature $T_{\mathrm{p}}$ and polarization time $\tau_{\mathrm{p}}$ ). The model of space-heterogeneous charge is proposed under assumption of blocking electrodes.

\section{Results}

Figures 1 and 2 illustrate in the $\ln \sigma-10^{3} / T$ coordinates the typical temperature dependence of the direct conductivity of the CGGG, CGG and AWO crystals. At the detailed consideration of the $\ln \sigma(1 / T)$ curves, they can be approximated by several linear segments. The activation energy values $E_{\text {a }}$ for each crystal and for the corresponding linear segments of $\ln \sigma(1 / T)$ curves are presented in Table I.

As Figs. 1-2 show, the $\sigma(T)$ curves are characterized by the low-temperature (LT) "structural-sensitivity" and high-temperature (HT) segments. For the LT and HT
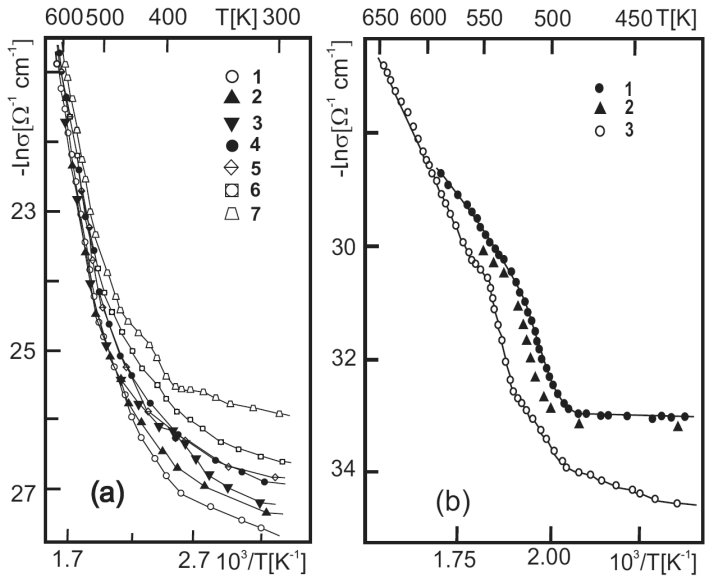

Fig. 1. Temperature dependences of electrical conductivity in direct external applied field for the CGGG (a) and CGG (b), in the air. The curves $1 \mathrm{a}$ and $2 \mathrm{a}-$ undoped CGGG (as electrical contacts correspondingly aquadag and Ag-paste are used); 3a - sample under high-thermal treatment in vacuum; 4a - CGGG:Cr; 5a - CGGG:Co; 6a - CGGG:Mn; 7a - CGGG:Er. The curve $1 \mathrm{~b}$ - measurement of CGG (z-cut); $2 \mathrm{~b}$ - the same measurement in vacuum; $3 \mathrm{~b}-x-y$-cut.

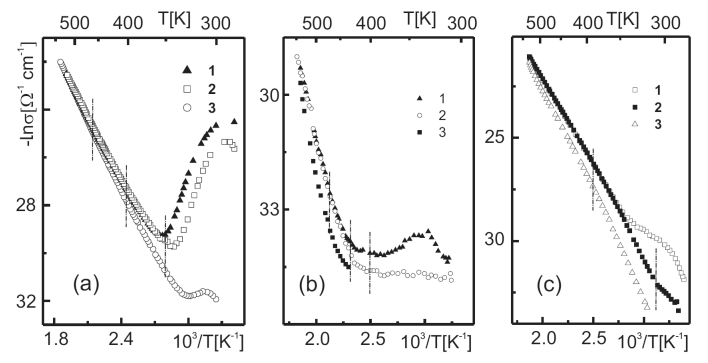

Fig. 2. Temperature dependences of electrical conductivity for PbWO (a), CaWO (b) and CdWO (c) crystals in the air, at direct applied electrical field. The curves 1a, b, c are obtained upon first heating; 2a, b, c - measurements upon second heating; $3 \mathrm{a}, \mathrm{b}, \mathrm{c}$ - upon cooling. The vertical lines depict the ranges with different values of the activation energy.

ranges, the $E_{\mathrm{a}}$ values are rather different. At low tem-

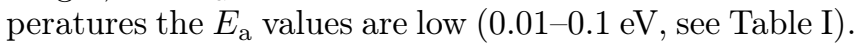
The $\sigma(T)$ curves in LT range are strongly dependent on: doped impurities, HT treatment (Fig. 1a), crystallographic axis in non-cubic crystals, prehistory of the specimens (Figs. 2a-c), and other experimental conditions. The HT region is less sensitive to the above mentioned factors. Here, the $E_{\mathrm{a}}$ values not exceed $2.51 \mathrm{eV}$ which is observed for CGG $(x-y$-cut) in the temperature range 530-560 K (Table I).

Among the AWO compounds the most intensive relaxation processes (Fig. 2a) are observed for the PbWO crystal in the LT range (290-350 K). The CaWO crystal in HT region is characterized by the highest $E_{\text {a }}$ value $(1.08 \mathrm{eV})$ relatively to other tungstates (see Table I). 
TABLE I

The values of activation energies $\left(E_{\mathrm{a}}\right)$ of the complex oxide crystals conductivity under direct external field for different temperature regions.

\begin{tabular}{|c|c|c|c|}
\hline \multicolumn{2}{|c|}{ Crystal } & $T[\mathrm{~K}]$ & $E_{\mathrm{a}}[\mathrm{eV}]$ \\
\hline \multirow{4}{*}{\multicolumn{2}{|c|}{ CGGG }} & $290-390$ & 0.10 \\
\hline & & $390-445$ & 0.28 \\
\hline & & $445-500$ & 0.55 \\
\hline & & $500-570$ & 1.22 \\
\hline \multirow{4}{*}{\multicolumn{2}{|c|}{ CGGG: $\mathrm{Cr}$ or $\mathrm{Co}$}} & $290-350$ & 0.12 \\
\hline & & $390-445$ & 0.25 \\
\hline & & $445-500$ & 0.53 \\
\hline & & $500-570$ & 1.18 \\
\hline \multirow{3}{*}{\multicolumn{2}{|c|}{ CGGG:Mn }} & $290-350$ & 0.06 \\
\hline & & $440-490$ & 9.35 \\
\hline & & $490-570$ & 1.08 \\
\hline \multirow{2}{*}{\multicolumn{2}{|c|}{ CGGG:Er }} & $450-490$ & 0.49 \\
\hline & & $490-570$ & 1.09 \\
\hline \multirow[t]{8}{*}{ CGG } & $Z$ & $290-450$ & 0.03 \\
\hline & & $500-530$ & 2.09 \\
\hline & & $530-560$ & 1.12 \\
\hline & & $290-450$ & 0.07 \\
\hline & $X-Y$ & $450-500$ & 0.21 \\
\hline & & $500-530$ & 0.85 \\
\hline & & $530-560$ & 2.51 \\
\hline & & $560-650$ & 1.48 \\
\hline \multirow{4}{*}{\multicolumn{2}{|c|}{$\mathrm{PbWO}$}} & $290-350$ & 0.23 \\
\hline & & $350-400$ & 0.66 \\
\hline & & $400-460$ & 0.72 \\
\hline & & $460-590$ & 0.85 \\
\hline \multirow{3}{*}{\multicolumn{2}{|c|}{$\mathrm{CaWO}$}} & $290-410$ & 0.02 \\
\hline & & $430-475$ & 0.74 \\
\hline & & $475-560$ & 1.08 \\
\hline \multirow{3}{*}{\multicolumn{2}{|c|}{ CdWO }} & $290-320$ & 0.42 \\
\hline & & $320-390$ & 0.82 \\
\hline & & $390-550$ & 0.71 \\
\hline
\end{tabular}

Figure 3 shows the frequency dependence of the real and imaginary parts of the conductivity at room temperature for the CGGG and CGG crystals. The values of $\sigma^{\prime}$ and $\sigma^{\prime \prime}$ parameters are linearized in the double logarithm coordinates. Therefore, the real and imaginary parts of the conductivity are proportional to frequency and correspond to the well-known exponential law from the Pollak theory [19]:

$$
\sigma(\omega) \sim A \omega^{n} .
$$

Here is $\omega=2 \pi f, A$ is a constant, and quantity $n$ is close to 1 .

From the inclination of the rectilinear segments of the $\log \sigma^{\prime}(\log f)$ and $\log \sigma^{\prime \prime}(\log f)$ dependences, we determined the values of the exponent $n_{1}$ and $n_{2}$ respectively,

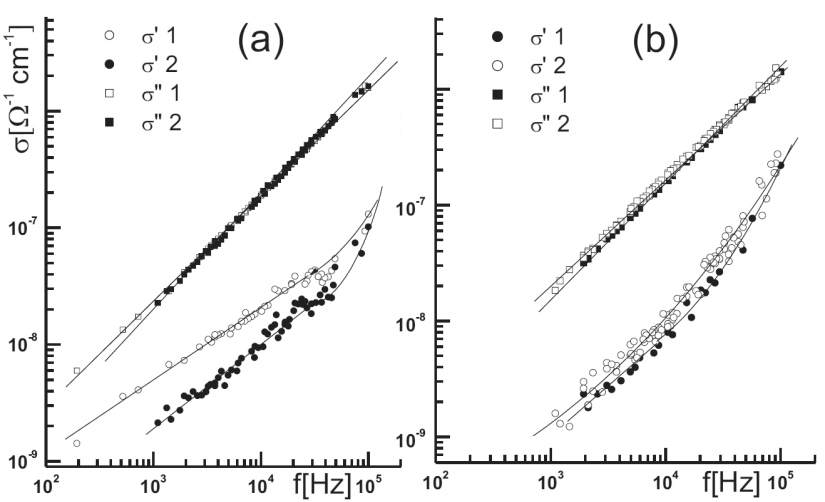

Fig. 3. Frequency dependences of real $\left(\sigma^{\prime}\right)$ and imaginary $\left(\sigma^{\prime \prime}\right)$ parts of electrical conductivity for CGG (a) and CGGG (b) crystals at $290 \mathrm{~K}$. The curve 1a corresponds to measurement for $z$-cut of CGG; $2 \mathrm{a}-x-y$-cut of CGG; 1b - undoped CGGG; 2b - CGGG:Nd.

which are close to 1 (see Table II). In the high-frequency region $\left(f>6 \times 10^{4} \mathrm{~Hz}\right)$ of $\lg \sigma^{\prime}(\log f)$ dependences, for the CGGG and CGG crystals is observed the deviation from linear law (3). The linear segment fit in this frequency region gives that $n_{3}$ lies within the 1-2 range. For both crystals $\log \sigma^{\prime \prime}(\log f)$ curves (Fig. 3) are lined at the whole frequency region. For the CGG crystal the crystallographic direction dependences of the $n_{1}, n_{2}$ and $n_{3}$ exponents (Table II) are observed. The $z$-cut CGG is distinguished by the relatively little value of exponent $n_{1}=0.64$. For the CGGG and CGGG:Nd crystals the data are similar (Fig. 3b).

TABLE II

The values of the exponents $\left(n_{1}, n_{2}\right.$, and $\left.n_{3}\right)$ in the law of frequency dependences of conductivity.

\begin{tabular}{l|c|c|c}
\hline \hline \multicolumn{1}{c|}{ Crystal } & $n_{1}$ & $n_{2}$ & $n_{3}$ \\
\hline CGGG & 0.99 & 0.94 & 1.80 \\
CGGG:Nd & 0.98 & 0.97 & 1.95 \\
CGG $(z$-cut) & 0.64 & 0.92 & 1.01 \\
CGG $(x-y$-cut) & 0.77 & 0.97 & 1.36
\end{tabular}

The frequency dependences of the dielectric constants $\varepsilon^{\prime}$ are demonstrated in Fig. 4. The $\varepsilon^{\prime}$ values decrease with increase in the frequency due to the polarization processes. For both crystals at frequencies $f>10^{3} \mathrm{~Hz}$ the quasi-stationary $\varepsilon^{\prime}$ values are registered.

For the relaxation processes study in the AWO crystals, the TSD currents were also measured. Figure 5 shows the typical TSD curves $I_{\mathrm{TSD}}(T)$ for the $\mathrm{PbWO}$ and CdWO crystals. For PbWO at temperatures 290-350 K, 350-380 $\mathrm{K}$ and $400-550 \mathrm{~K}$ the complex current maxima (Fig. 5) are registered. As it was shown previously in $[11,13]$ the maxima $I_{\mathrm{TSD}}(T)$ at $290-380 \mathrm{~K}$ are due to the dipolar relaxation. The maximum at $400-550 \mathrm{~K}$ is caused by the formation of space charge as a result of 


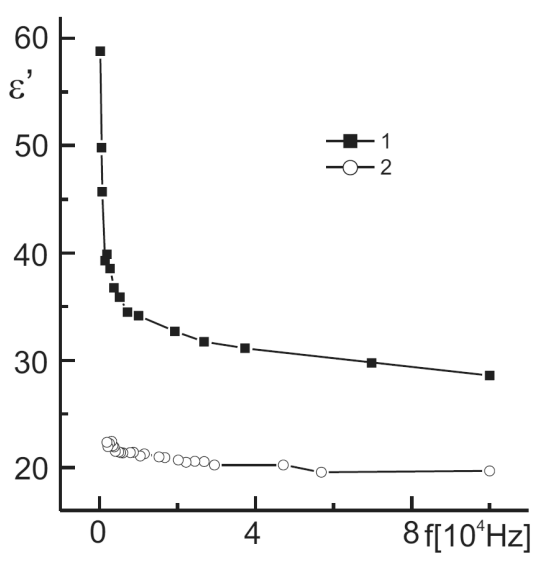

Fig. 4. The dielectric constant on frequency dependence for the $z$-cut of CGG (1) and CGGG (2) crystals at $290 \mathrm{~K}$.

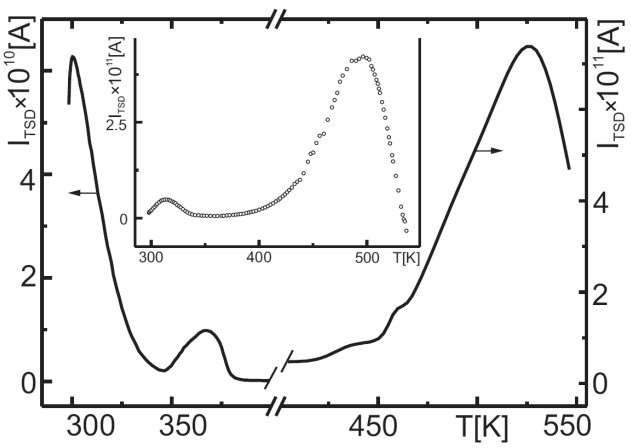

Fig. 5. The typical curves of thermal depolarization current of the PbWO and CdWO (in the inset) crystals. The polarization conditions of the sample are: $\mathrm{PbWO}$ $-T_{\mathrm{p}}=330 \mathrm{~K}$; CdWO $-T_{\mathrm{p}}=450 \mathrm{~K}$; in both cases tests were done at $U_{\mathrm{p}}=+50 \mathrm{~V}, \tau_{\mathrm{p}}=5 \mathrm{~min}$.

migration of equilibrium charge carriers and their localization. The active relaxation processes of dipolar nature (290-380 K) essentially influence the curve shape $\varepsilon(T)$ in this temperature region for the PbWO crystal [13].

The typical TSD spectrum for the CdWO crystal is shown in Fig. 5 (inset). The HT segment of thermogram for the CdWO crystal at $T_{\mathrm{p}}>450 \mathrm{~K}$ is substantially varied. The intense complex maximum of TSD with large half-width is formed. The TSD peak of CdWO in 290-340 K, as it was shown previously [13], corresponds to dipolar nature and the maximum in $400-550 \mathrm{~K}$ is created by spatially inhomogeneous distribution of space charge.

\section{Discussion and conclusions}

The CGGG, CGG and AWO crystals, as follows from the considered experimental data, are high-resistant materials. The point defects in these crystals are described by the Kröger-Vink model [20]. The temperature and frequency dependences of the conductivity (Figs. 1-3) testify about the mixed ion-electron type of electrotransfer. The complex polarization processes and interaction between the electrically active defects influence the conductivity (as it is shown in Figs. 4-5).

The $\sigma^{\prime}(f)$ and $\sigma^{\prime \prime}(f)$ curves of CGGG and CGG crystals are described by increasing law (3). This criterion corresponds to the hopping mechanism of the electronic part of conductivity and formation of small polarons. This conclusion is confirmed by $E_{\mathrm{a}}$ values (Table I) in the region $290-500 \mathrm{~K}$. The increasing $n$ values in the region $f>6 \times 10^{4} \mathrm{~Hz}$, due to the relation (3) can be connected with increase of the non-phonon (resonant) contribution [21] to the active conductivity of crystals.

The simplest dipole associates, which partly determine the dipolar polarization/depolarization (Fig. 5) in the studied crystals, are possibly the dipolons $[9,13]$, in other words the pairs of oppositely charged vacancies (cationic + anionic) with possible localization of charge carriers on such structural defects. The charge exchange of these centres is realized by the hopping-like manner. It partly causes the frequency dependence of conductivity. The complex defects are formed on the bases of dipolons. Their association/dissociation gives the contribution to the relaxation processes. In these phenomena the role of the edge dislocations, which have the charge and may interact with vacancies, is not excluded. Thus, in AWO crystals the dislocations density is about $10^{3}-10^{6} \mathrm{~cm}^{-2}$ $[22,23]$.

In the crystals with deviation from stoichiometry the vacancy defects dominate [24] and have tendency to group forming and even to the formation of the ordered structures or clusters [8]. At the same time, in electrotransfer the participation of noncontrolled impurities is possible. The phenomenological brief analysis of the obtained data testify: for establishing the final models of dipole associates, determination of dominating type of electrically active defects, ascertainment of regularities of the complex formation and electrotransfer mechanisms in the studied crystals additional investigations are required.

\section{References}

[1] The Physics and Spectroscopy of Laser Crystals. Ed. A.A. Kaminskii, Nauka, Moscow 1986 (in Russian).

[2] A.A. Kaminskii, E.L. Belokoneva, B.V. Mill, Yu.V. Pisarevskii, S.E. Sarkisov, I.M. Silvestrova, A.V. Butashin, G.G. Khodzhabagyan, Phys. Status Solidi A 86, 345 (1984).

[3] M. Nikl, V.V. Laguta, A. Vedda, Phys. Status Solidi B 245, 1701 (2008).

[4] V.D. Ryzhikov, V.G. Bondar', L.L. Nagornaya, Functional Materials for Science and Engineering. Ed. V.P. Seminozhenko, Institute for Single Crystals, Kharkov 2001, p. 91 (in Russian).

[5] P. Lecoq, in: Proc. Int. Conf. Inorganic Scintillators and their Applications. SCINT 95, Eds. P. Dorenbos, C.W.E. van Eijk, Delft University Press, Delft, The Netherlands 1996, p. 52. 
[6] V.I. Dormenev, A.N. Annenkov, A.Ye. Borisevich, G.Yu. Drobyshev, M.V. Korzhik, K.B. Rudov, Dev. Techn. Exp. 2, 71 (2005) (in Russian).

[7] Yu.S. Kuz'minov, Kristallografiya 51, 533 (2006) (in Russian).

[8] S.F. Burachas, A.A. Vasil'ev, M.S. Ippolitov, V.I. Man'ko, Yu.A. Savel'ev, G. Tamulaitis, Crystallogr. Reports 52, 1088 (2007).

[9] A.E. Nosenko, V.N. Shevchuk, Radiat. Eff. Def. Solids. 134, 251 (1995).

[10] A.E. Nosenko, V.N. Shevchuk, Phys. Solid State 39, 775 (1997).

[11] V.N. Shevchuk, I.V. Kayun, Phys. Solid State 45, 1898 (2003)

[12] V.N. Shevchuk, I.V. Kayun, Functional Materials 13 , 584 (2006).

[13] V.N. Shevchuk, I.V. Kayun, Radiat. Measurements 42, 847 (2007).

[14] B.V. Mill, B.A. Maximov, Yu.V. Pisarevsky, N.P. Danilova, A. Pavlovskaya, Sh. Verner, Yu. Shnaider, Kristallografiya 49, 65 (2004) (in Russian).

[15] V.N. Shevchuk, I.V. Kayun, Phys. Solid State 47, 632 (2005).
[16] V.N. Shevchuk, I.V. Kayun, Functional Materials 10 , 220 (2003).

[17] V.N. Shevchuk, I.V. Kayun, J. Phys. Stud. 13, 1703-1 (2009) (in Ukrainian).

[18] Yu.A. Gorochovatsky, G.A. Bordovsky, Thermally Activational Current Spectroscopy of High-Resistance Semiconductors and Dielectrics Nauka, Moskow 1991 (in Russian).

[19] M. Pollak, Phys. Rev. A 133, 564 (1964).

[20] F.A. Kröger, The Chemistry of Imperfect Crystals, North-Holland, Amsterdam, New York 1964.

[21] I.V. Klatskina, I.S. Shlimak, Fiz. Tekh. Poluprovodn. 12, 134 (1978) (in Russian).

[22] S. Ganesamoorthy, I. Bhaumik, A.K. Karnal, V.K. Vadhawan, J. Cryst. Growth 264, 320 (2004).

[23] B.P. Nazarenko, V.N. Baumer, E.F. Dolzhenkova, M.B. Kosmyna, Inorganic Materials 41, 1114 (2005).

[24] A.I. Gusev, Non-Stoichiometry, Disorder, Short-Range and Long-Range Order in Solids, Fizmatlit, Moscow 2007 (in Russian). 